\section{Rheb GTPase is a direct target of TSC2 GAP activity and regulates $\mathrm{mTOR}$ signaling}

\author{
Ken Inoki, ${ }^{1,4}$ Yong $\mathrm{Li}^{1,4}$ Tian $\mathrm{Xu}^{1,}$ and \\ Kun-Liang Guan 1,2,5 \\ ${ }^{1}$ Department of Biological Chemistry, ${ }^{2}$ Institute of \\ Gerontology, University of Michigan Medical School, \\ Ann Arbor, Michigan 48109, USA; ${ }^{3}$ Howard Hughes Medical \\ Institute, Department of Genetics, Yale University School \\ of Medicine, New Haven, Connecticut 06536, USA
}

Tuberous sclerosis complex (TSC) is a genetic disease caused by mutation in either TSC1 or TSC2. The TSC1 and TSC2 gene products form a functional complex and inhibit phosphorylation of $\mathrm{S} 6 \mathrm{~K}$ and 4EBP1. These functions of TSC1/TSC2 are likely mediated by mTOR. Here we report that TSC2 is a GTPase-activating protein (GAP) toward Rheb, a Ras family GTPase. Rheb stimulates phosphorylation of S6K and 4EBP1. This function of Rheb is blocked by rapamycin and dominant-negative mTOR. Rheb stimulates the phosphorylation of mTOR and plays an essential role in regulation of $S 6 K$ and 4EBP1 in response to nutrients and cellular energy status. Our data demonstrate that Rheb acts downstream of TSC1/TSC2 and upstream of $\mathrm{mTOR}$ to regulate cell growth.

Received May 6, 2003; revised version accepted June 2, 2003.

Tuberous sclerosis complex (TSC) is characterized by hamartoma formation in a wide range of tissues and is a genetic disorder caused by mutations in either the TSC1 or the TSC2 gene (Kwiatkowski 2003). The most serious clinical complications are mental retardation, epilepsy, and autism caused by tumor growth in the brain (Gomez 1991). Other symptoms include renal dysfunction, dermatological abnormalities, and heart problems. Heterozygous deletions of either TSC1 or TSC2 produce similar phenotypes and increase tumor incidence particularly in the kidney, whereas homozygous deletion of either gene is embryonic lethal (Au et al. 1998; Kobayashi et al. 2001). Genetic data from Drosophila show that TSC1 and TSC2 negatively regulate cell growth and cell size (Gao and Pan 2001; Potter et al. 2001; Tapon et al. 2001).

Recent studies have established that TSC1/TSC2 inhibits phosphorylation of the ribosomal S6 kinase (S6K) and the eukaryotic initiation factor 4E-binding protein (4EBP1), two key regulators of translation (Goncharova et al. 2002; Inoki et al. 2002; Manning et al. 2002; Tee et al. 2002). Phosphorylation of S6K and 4EBP1 enhances translation (Gingras et al. 1999). How TSC1/TSC2 regu-

[Keywords: TSC2; Rheb; mTOR; S6K; GAP; tuberous sclerosis complex] ${ }^{4}$ These authors contributed equally to this work. ${ }^{5}$ Corresponding author.

E-MAIL kunliang@umich.edu; FAX (734) 763-4581.

Article published online ahead of print. Article and publication date are at http://www.genesdev.org/cgi/doi/10.1101/gad.1110003. lates the phosphorylation of S6K and 4EBP1 is a key question yet to be answered. The mammalian target of rapamycin (mTOR) is directly responsible for phosphorylation of both S6K and 4EBP1 (Brown et al. 1995; Hara et al. 1998). Recent studies have suggested that TSC1/ TSC2 acts through $\mathrm{mTOR}$ to regulate the phosphorylation of S6K and 4EBP1 (Gao et al. 2002; Inoki et al. 2002; Tee et al. 2002). Consistent with this model is that TSC1 and TSC2 are important for cellular nutrient response, which requires the function of mTOR (Gao et al. 2002). However, it is unclear how TSC1/TSC2 inhibits mTOR activity.

The C-terminal region of TSC2 displays significant homology to the Rap GTPase-activating protein (GAP; The European Chromosome 16 Tuberous Sclerosis Consortium 1993). In fact, GAP activity of TSC2 toward Rap1 and Rab5 had previously been reported (Wienecke et al. 1995; Xiao et al. 1997). However, the reported GAP activity was extremely low, and the functional significance is unclear. Rheb is a small GTPase initially isolated as a Ras homolog enriched in brain (Yamagata et al. 1994) and is widely expressed. Rheb shares higher sequence identity with Ras than with Rho family members. The biological function of mammalian Rheb is unclear. Conflicting studies report that Rheb both inhibits and activates the Raf-MAP kinase pathway (Clark et al. 1997; Yee and Worley 1997). Interestingly, mutation of the Rheb gene in Schizosaccharomyces pombe produces a phenotype similar to nutrient starvation, indicating that Rheb is possibly involved in nutrient signaling (Mach et al. 2000).

In this report, we provide direct biochemical data demonstrating that TSC2 has GAP activity toward Rheb in vitro. Furthermore, we show that TSC2 regulates RhebGTP levels in vivo. We show that one of the roles of Rheb is to stimulate phosphorylation of S6K and 4EBP1, two of the best characterized cellular downstream targets of TSC1/TSC2. Both the effector domain and the GTP binding are essential for Rheb function. The ability of Rheb to stimulate S6K phosphorylation requires the function of $\mathrm{mTOR}$, indicating that $\mathrm{mTOR}$ acts downstream of Rheb. Consistent with this is that Rheb stimulates the phosphorylation of mTOR on serine residue 2448. Furthermore, our data support that Rheb plays an important role in cellular responses to energy limitation and nutrient starvation. Together, this study provides a model that Rheb is a direct downstream target of TSC2 and acts upstream of mTOR to regulate translation and cell growth.

\section{Results and Discussion}

\section{TSC2 stimulates GTP hydrolysis of Rheb}

The C-terminal region of TSC2 contains a putative GAP domain with significant homology to RapGAP (The European Chromosome 16 Tuberous Sclerosis Consortium 1993; Scheffzek et al. 1998). However, the precise physiological and biochemical functions of the putative TSC2 GAP domain have not been demonstrated. Interestingly, a high frequency of TSC-associated mutations occurs in the C-terminal putative GAP domain of TSC2, indicating the GAP domain may be important for TSC2 function (Jin et al. 1996; Momose et al. 2002; Kwiatkowski 
Inoki et al.

2003). To study the biochemical functions of TSC2, we expressed and purified the GAP domain of TSC2 in Escherichia coli and tested for GAP activity toward the Ras subfamily GTPases (Ras, Rap, TC21, and Rheb) and the Rho family GTPases (Rac and Cdc42). Our in vitro assays failed to detect significant GAP activity, whereas the positive control of RasGAP1 showed activity toward Ras (data not shown). It is worth noting that the catalytic arginine residue essential for GAP activity in the Rap GAP family is not conserved in TSC2 (Fig. 1A), suggesting that TSC2 may have no GAP activity (Scheffzek et al. 1998). It is also possible that TSC2 has GAP activity, but a different arginine or a completely different catalytic mechanism may be used.

To further test for GAP activity of TSC2, we expressed TSC2 together with TSC1 in HEK293 cells. The TSC1/ TSC2 complex was purified by immunoprecipitation, and in vitro GAP activity was determined using various small GTPases. We made particular efforts on Rheb because deletion of Rheb in $S$. pombe produces phenotypes similar to nutrient starvation (Mach et al. 2000). We found that the TSC1/TSC2 complex stimulated the GTP hydrolysis of Rheb (Fig. 1B,C) but not Ras. The GAP activity of TSC2 requires the full-length protein because neither the $\mathrm{N}$-terminal nor the $\mathrm{C}$-terminal region, which contains the GAP homology domain, displayed activity toward Rheb (Fig. 1D). To exclude the possibility that the GAP activity detected in the TSC1/TSC2 immunoprecipitation is due to another coprecipitated GAP, we made TSC2-R1701Q and TSC2-R1703Q mutants, which are found in TSC patients, and detected no GAP activity (data not shown). Therefore, we conclude that TSC2 is an Rheb GAP. The lack of GAP activity of TSC2C could be due to the requirement of residues in the $\mathrm{N}$-terminal region of TSC2 for GAP activity or incorrect folding of TSC2C. The ability of TSC2 to stimulate GTPase activity of Rheb is very interesting because TSC2 does not have the conserved active-site arginine found in other related RapGAPs and Rheb is an unusual GTPase (Fig. 1A). Almost all Ras family GTPases contain a conserved glycine residue at the position equivalent to codon 12 in Ras (Lowy and Willumsen 1993). Mutation of this glycine to almost any residue, including valine found in many cancers, activates Ras family GTPases. The RasV12 mutant is known to be resistant to down-regulation by RasGAP (Trahey and McCormick 1987). Interestingly, Rheb has an arginine residue at the corresponding position codon 12 and has a much lower basal GTPase activity than Ras (data not shown). However, the GTPase activity of Rheb can be effectively stimulated by TSC1/TSC2. Therefore, Rheb and TSC2 are unusual GTPases and GAPs, respectively.

To determine whether TSC1 is required for the GAP activity of TSC2, HA-TSC2 alone was expressed in HEK293 cells and immunoprecipitated. The precipitated TSC2, which may also coimmunoprecipitate a small amount of endogenous TSC1, showed significant GAP activity toward Rheb (Fig. 1E). In contrast, immunopre-

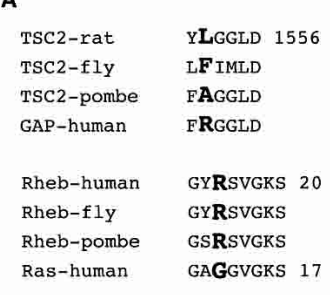

D

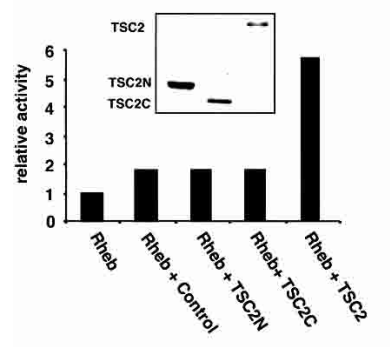

B

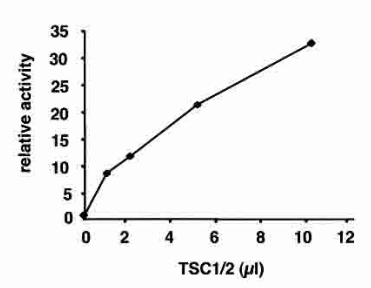

E

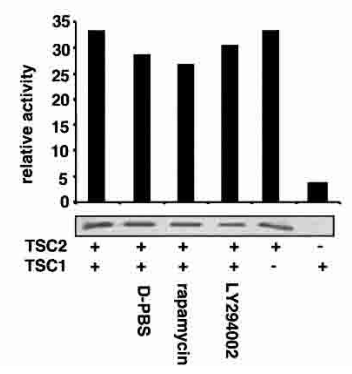

C

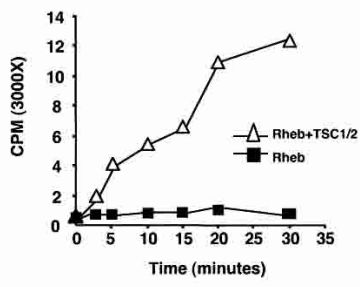

$\mathbf{F}$

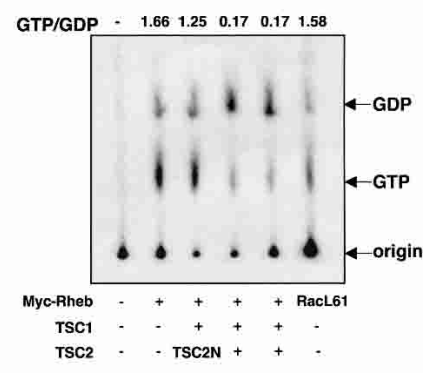

Figure 1. TSC2 has GAP activity toward Rheb. (A) Rheb and TSC2 are an unusual GTPase and GAP. The catalytic active-site arginine (the residue in bold) in RapGAP is not conserved in TSC2. Rheb contains an arginine residue (the residue in bold) at the position corresponding to codon 12 of Ras, which has a glycine. Numbers indicate positions of the last residues. $(B)$ Immunoprecipitated TSC1/TSC2 stimulates GTP hydrolysis of Rheb. Increasing amounts (in microliters) of immunoprecipitated TSC1/TSC2 were incubated with GST-Rheb at room temperature for $20 \mathrm{~min}$. Release of free ${ }^{32} \mathrm{P}$-phosphate was measured by radioactive counting. Background activity of a control immunoprecipitation was subtracted. The basal GTPase activity of Rheb was arbitrarily set as $1 .(C)$ Time-dependent GTP hydrolysis of Rheb stimulated by TSC1/TSC2. GTP hydrolysis was determined in the absence $(\mathbf{\square})$ and presence $(\triangle)$ of TSC1/TSC2. $(D)$ Neither the N-terminal nor the C-terminal region of TSC2 has GAP activity toward Rheb. Two truncated TSC2 constructs (TSC2N, amino acids 1-1007; TSC2C, amino acids 1008-1765) were coexpressed with TSC1 and immunoprecipitated and assayed for GAP activity in vitro. The amount of HA-TSC2 used in this assay is equivalent to $1 \mu \mathrm{L}$ in $B$. The expression levels of these proteins were determined by Western blot. (E) TSC2, but not TSC1, has GAP activity. Transfected HEK293 cells were untreated or treated with D-PBS, rapamycin $(20 \mathrm{nM})$, or LY294002 $(50 \mu \mathrm{M})$ for 30 min as indicated. The relative amount of TSC2 used in the GAP assay was determined by Western blot and is shown below each bar. (F) TSC1/TSC2 decreases the Rheb-GTP levels in vivo. Myc-Rheb was transfected in HEK293 cells and labeled with ${ }^{32} \mathrm{P}$-phosphate. Myc-Rheb was immunoprecipitated, and the bound nucleotides were eluted and resolved on a cellulose plate. Cotransfection with TSC1 and TSC2 are indicated. Myc-RacL61 was included as a control. The ratio of GTP/GDP was calculated by the formula GTP counts/3 divided by GDP counts/2, and indicated on top of each lane. 
cipitated TSC1 showed a very low level of GAP activity toward Rheb possibly due to coprecipitation of endogenous TSC 2 protein. These observations indicate that TSC1 stabilizes TSC2 in vivo, but is not directly required for GAP activity. TSC1/TSC2 was also immunoprecipitated under various conditions, including treatments with rapamycin, LY294002, and D-PBS, which are all known to inhibit phosphorylation of S6K and 4EBP1. We observed that these treatments did not directly affect the GAP activity of immunoprecipitated TSC1/TSC2 (Fig. 1E).

The effect of TSC2 on Rheb GTP levels was also determined in vivo. The nucleotide-binding status of Rheb was determined by in vivo labeling with ${ }^{32} \mathrm{P}$-phosphate. Interestingly, Rheb contains a high basal GTP level (Fig. $1 F)$. The high GTP level of wild-type Rheb is consistent with it having an arginine at a position equivalent to codon 12 of Ras (Fig. 1A). Expression of TSC1/TSC2 decreased the GTP/GDP ratio of cotransfected Rheb 10fold (Fig. 1F). As a control, expression of the N-terminal domain of TSC2 had no significant effect on the GTP level of Rheb. These data provide in vivo evidence that TSC2 is an Rheb GAP.

\section{Rheb stimulates the phosphorylation of S6K and $4 E B P 1$}

If Rheb is a key downstream target of TSC2, Rheb should stimulate the phosphorylation of S6K and 4EBP1. Expression of the wild-type Rheb induced a dramatic increase in S6K phosphorylation and mobility shift of 4EBP1 (Fig. 2A). Rheb also increased the phosphorylation of endogenous S6K in transfected HEK293 cells. In contrast, Rheb did not stimulate ERK phosphorylation (data not shown). The high activity of wild-type Rheb is consistent with it being predominantly GTP bound (Fig. 1F). Rheb also stimulates S6K phosphorylation in a dose-dependent manner (Fig. 2B).
Five residues between 38 and 42 in the effector domain of Rheb were mutated to alanines to produce Rheb5A. We found that mutation of the effector domain completely abolished the ability of Rheb to stimulate S6K phosphorylation (Fig. 2C). We also tested constitutively active Rheb (RhebL64) and observed that RhebL64 is approximately two to three times more active than the wild type (Fig. 2C). In an attempt to create a dominantnegative mutant, we made an RhebN20. Surprisingly, RhebN20 does not show any dominant-negative effect nor does it stimulate S6K (Fig. 2C). A similar dominantnegative mutant of RasN17 is effective in blocking Ras function through sequestration of the Ras guanine nucleotide exchange factor (GEF; Lowy and Willumsen 1993). This observation suggests that Rheb may not need a GEF because of its high basal GTP level and that GTP binding is required to stimulate $\mathrm{S} 6 \mathrm{~K}$ phosphorylation.

To determine the specificity of Rheb, several other GTPases were examined, including Cdc42, Rap1, RhoA, and Rab5. Constitutively active mutants of these GTPases fail to stimulate S6K phosphorylation (Fig. 2D). These results demonstrate that Rheb is specific to stimulate S6K phosphorylation. We tested the effect of TSC1 or TSC2 alone on S6K phosphorylation. Expression of TSC2, but not TSC1, significantly decreased the basal phosphorylation of cotransfected S6K (Fig. 3E), consistent with the fact that TSC2 alone has GAP activity. The ability of TSC2 but not TSC1 to inhibit S6K indicates that TSC2 is the active component of the TSC1/TSC2 complex, whereas TSC1 may have regulatory functions, such as localization and stabilization. Furthermore, these observations suggest that inhibition of endogenous Rheb by TSC2 decreases S6K phosphorylation, thus supporting the importance of Rheb in S6K regulation.

\section{TSC1/TSC2 inhibits Rheb function}

The functional relationship between TSC1/TSC2 and Rheb was examined in vivo. Cotransfection of TSC1/

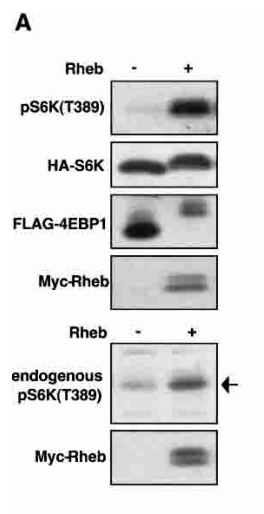

B
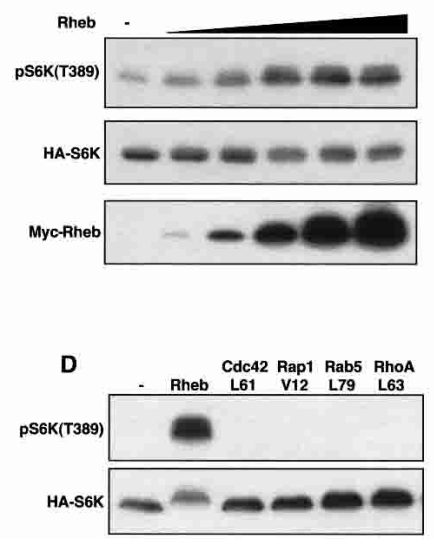

C
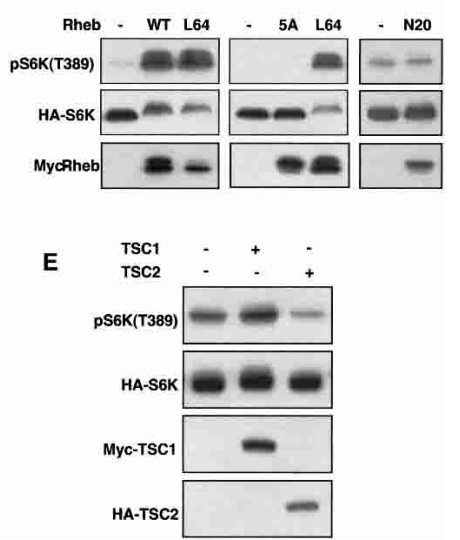

Figure 2. Rheb stimulates phosphorylation of S6K and 4EBP1. (A) Rheb stimulates phosphorylation of S6K and 4EBP1. HA-S6K and Flag-4EBP1 were transfected into HEK293 cells in the presence or absence of Myc-Rheb (100 ng). Phosphorylation of S6K was determined by phosphospecific antibody, pS6K(T389), whereas phosphorylation of 4EBP1 was determined by mobility shift (upper panels). Phosphorylation of endogenous S6K was also determined (lower panels). (B) Rheb stimulates phosphorylation of S6K in a dose-dependent manner. HA-S6K (15 ng) was cotransfected with 0, 2, 5, 20, 50, and $100 \mathrm{ng}$ of Myc-Rheb. $(C)$ The effector domain of Rheb is required to stimulate S6K phosphorylation. HA-S6K was cotransfected with wild-type Rheb (100 ng), RhebL64 (40 ng), Rheb-5A (200 ng), and RhebN20 (300 ng). The relative expression of Rheb mutants is also shown. (D) Stimulation of S6K phosphorylation by different GTPases. Constitutively active mutants (100 ng for each) of Cdc42, Rap1, Rab5, RhoA, and the wild-type Rheb (40 ng) were used to stimulate phosphorylation of S6K. (E) TSC2, but not TSC1, inhibits S6K phosphorylation. HA-S6K was coexpressed with either Myc-TSC1 or HA-TSC2. Phosphorylation of S6K was determined. 
Inoki et al.
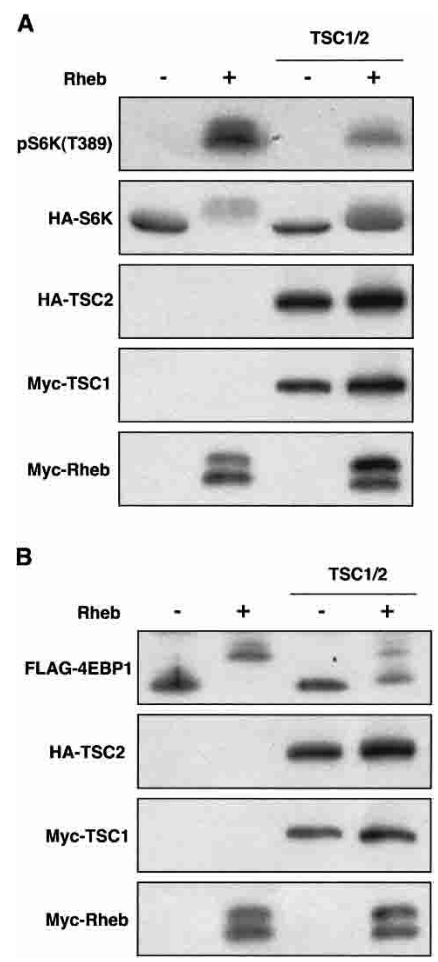

Figure 3. TSC1/TSC2 inhibits Rheb function in vivo. (A) TSC1/ TSC2 inhibits the ability of Rheb to induce S6K phosphorylation. $(B)$ TSC1/TSC2 inhibits the ability of Rheb to induce 4EBP1 phosphorylation.

TSC2 suppressed the ability of Rheb to stimulate S6K (Fig. 3A). Similar results were observed with 4EBP1 (Fig. 3B). These data further support the idea that TSC2 acts as a GAP toward Rheb.

\section{mTOR acts downstream of Rheb}

Previous studies have suggested that mTOR acts downstream of TSC2 (Gao et al. 2002; Inoki et al. 2002; Tee et al. 2002). To determine the relationship between Rheb and mTOR, the effect of rapamycin was investigated. Treatment of cells with rapamycin completely blocked the stimulatory effect of Rheb on S6K phosphorylation (Fig. 4A), indicating that Rheb functions upstream of mTOR. To further test the functional relationship between Rheb and mTOR, a kinase-inactive mutant of mTOR was coexpressed with Rheb. We found that the dominant-negative mTOR-KD significantly blocked the phosphorylation of S6K by Rheb (Fig. 4B). We had previously observed that TSC1/TSC2 inhibits the phosphorylation of S2448 of mTOR (Inoki et al. 2002; Kenerson et al. 2002). Coexpression of Rheb caused a significant increase of mTOR S2448 phosphorylation, whereas phosphorylation of Akt was not affected (Fig. 4C), indicating that Rheb activates mTOR.

\section{Rheb is involved in multiple signaling pathways that regulate $S 6 \mathrm{~K}$}

Phosphorylation of S6K is regulated by numerous cellular signals including nutrients (Proud 2002). The functional significance of Rheb in nutrient signaling was analyzed. In Rheb-transfected HEK293 cells, nutrient starvation (treatment with D-PBS that contains glucose, but not amino acids) induced a minor dephosphorylation of S6K (Fig. 4A). In contrast, nutrient starvation inhibited S6K phosphorylation in RasV12-transfected cells (Fig. 4A). These results demonstrate that Rheb plays an important role in the nutrient signaling pathway, but Ras is not directly involved in nutrient signaling. We also examined the effect of osmotic stress by sorbitol, energy depletion by 2-deoxyglucose (2-DG), and inhibition of phospholipase D by 1-butanol. These treatments are
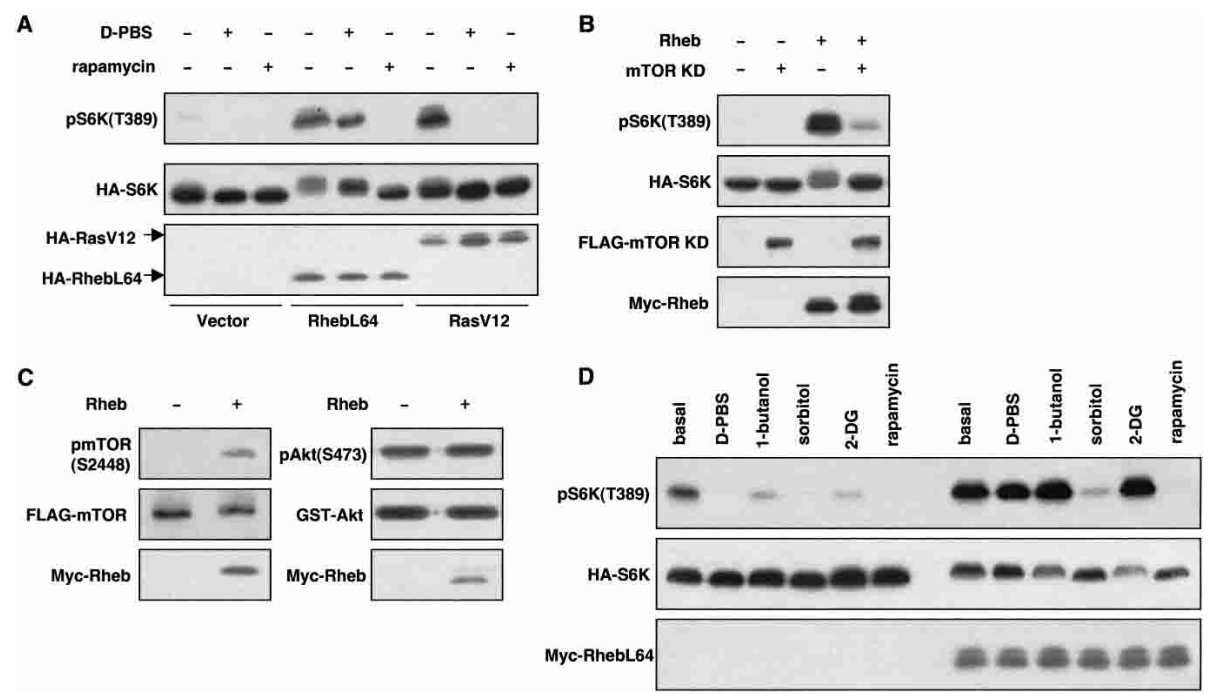

Figure 4. Rheb functions upstream of mTOR and is involved in response to various signals. $(A)$ Rheb acts downstream of nutrient signals and upstream of mTOR. HA-S6K was cotransfected with RhebL64 or RasV12 as indicated. Cells were treated with rapamycin or D-PBS for 30 min. Phosphorylation of S6K was determined. $(B)$ Kinase inactive mTOR KD blocks Rheb-induced S6K phosphorylation. $(C)$ Rheb stimulates the phosphorylation of mTOR. Flag-mTOR or GST-Akt was cotransfected with Rheb and immunoprecipitated. (Left) Phosphorylation of mTOR was detected by the anti-phospho mTOR (S2448) antibody. (Right) Phosphorylation of Akt was monitored by the anti-phospho Akt (S473) antibody. $(D)$ Role of Rheb in S6K regulation by various signaling pathways. HA-S6K was transfected alone (left half) or together with RhebL64 (right half). Cells were treated with D-PBS, 1-butanol $(0.3 \%)$, sorbitol $(600 \mathrm{mM})$, 2-DG (25 mM), or rapamycin (20 nM) for $30 \mathrm{~min}$ as indicated. 
known to induce S6K dephosphorylation (Fig. 4D; Dennis et al. 2001; Fang et al. 2001; Desai et al. 2002). Rheb significantly blocked the effect of 2-DG and 1-butanol, but had little effect on S6K dephosphorylation induced by sorbitol (Fig. 4D). These data suggest that Rheb acts in the cellular energy-sensing pathway and the phospholipase D pathway, but is not directly involved in the osmotic stress pathway. Consistently, TSC1/TSC2 also plays an important role in regulation of $\mathrm{S} 6 \mathrm{~K}$ in response to cellular energy depletion (data not shown).

The importance of TSC1/TSC2 in cell growth regulation has been well established (Potter and $\mathrm{Xu}$ 2001). The major cellular function of TSC1/TSC2 is to inhibit the phosphorylation of S6K and 4EBP1, likely through mTOR. This study provides a significant advance in the understanding of the molecular mechanism of how TSC1/TSC2 regulates the phosphorylation of S6K and $4 \mathrm{EBP}-1$. Based on both in vitro and in vivo biochemical experiments, we conclude that TSC2 is an Rheb-specific GAP and Rheb is a key downstream target of TSC1/ TSC2 (Fig. 5). Rheb is closely related to Ras and is an unusual GTPase in that it is predominantly GTP bound in cells. The biological function of Rheb has not been clearly defined. Our studies support that Rheb promotes cell growth by stimulating phosphorylation of S6K and 4EBP1, but has no role in ERK activation.

Inhibition of Rheb by TSC1/TSC2 is likely to be critical for the function of these two tumor suppressor proteins because Rheb potently regulates the phosphorylation of S6K and 4EBP1. Interestingly, mutation of the Rheb ortholog in S. pombe displays phenotypes characteristic of nutrient starvation (Mach et al. 2000). Our data are consistent with the genetic observations made in $S$. pombe and support the notion that Rheb plays an essential role in nutrient signaling. $S$. pombe also contains TSC1- and TSC2-related molecules (Matsumoto et al. 2002). We have also shown that TSC2 is an unusual GAP because it lacks the conserved arginine found in other Rap family GAPs. Yet it has very high and selective GAP activity toward Rheb. Future studies to elucidate the catalytic mechanism of TSC2 on Rheb-GTP hydrolysis will provide new insights into how this unusual GTPase and GAP work.

Our studies present a model in which Rheb is involved in S6K regulation in response to nutrients and energy levels (Fig. 5). In contrast, osmotic stress regulates S6K independently of Rheb. A major function of Rheb is to stimulate mTOR; however, we did not detect a significant association between Rheb and mTOR, suggesting that Rheb may activate mTOR indirectly. Future efforts

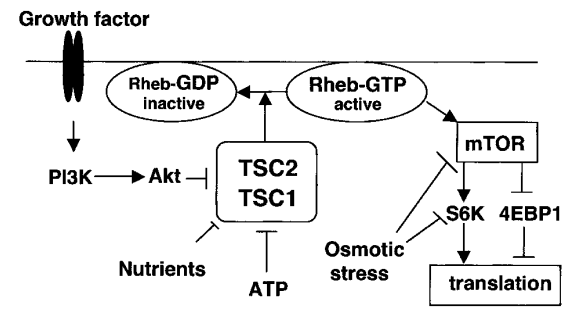

Figure 5. A proposed model of Rheb functions downstream of TSC1/TSC2 and upstream of mTOR. TSC2 acts as a GAP to inactivate Rheb by directly stimulating GTP hydrolysis. Rheb stimulates mTOR. Nutrient and cellular energy status signals through Rheb, whereas osmotic stress signals independently of Rheb. will focus on how Rheb stimulates mTOR. Our model predicts that TSC2 acts through Rheb to regulate translation (Fig. 5). It suggests that Rheb positively regulates cell growth and plays an important role in the development of TSC. Therefore, inhibition of Rheb could be a potential target for the treatment of TSC and cancer.

\section{Materials and methods}

\section{Antibodies and plasmids}

Anti-phospho S6K (T389), anti-mTOR, and anti-phospho mTOR (S2448 antibodies were from Cell Signaling Inc., and anti-Myc antibodies were from Santa Cruz Biotechnology. Anti-HA and anti-Flag were purchased from Covance and Sigma, respectively. TSC1 and TSC2 constructs were described previously (Inoki et al. 2002). The HA-tagged S6K1 ( $\alpha$ II) construct was generously provided by J. Blenis (Harvard University, Cambridge, MA) and Flag-tagged mTOR and kinase inactive mTOR (mTORKD) were from S. Schreiber (Harvard University, Cambridge, MA). Rab5 is a gift of G. Li (University of Oklahoma, Health Sciences Center, Oklahoma City, OK). All other DNA constructs including Ras, Rheb, Rap1, Rac, Cdc42, RhoA, 4E-BP1, Akt, and ERK2 were laboratory stocks. All mutant constructs of TSC2 and Rheb were created by PCR mutagenesis and verified by DNA sequencing.

Cell culture, transfection, and immunoprecipitation

HEK293 cells were seeded and maintained in Dulbecco's modified Eagle's medium (DMEM) containing 10\% fetal bovine serum (FBS). Transfection was performed using Lipofectamine Reagent (Invitrogen) following the manufacturer's instructions. Cells were lysed in lysis buffer $(10$ $\mathrm{mM}$ Tris- $\mathrm{HCl}$ at $\mathrm{pH} 7.5,100 \mathrm{mM} \mathrm{NaCl}, 1 \% \mathrm{NP}-40,1 \%$ Triton X-100, 50 $\mathrm{mM} \mathrm{NaF}, 2 \mathrm{mM}$ EDTA, $1 \mathrm{mM}$ PMSF, $10 \mu \mathrm{g} / \mathrm{mL}$ leupeptin, $10 \mu \mathrm{g} / \mathrm{mL}$ aprotinin) and immunoprecipitated with the indicated antibodies and protein G-Sepharose beads. Immunocomplexes were subjected to SDSPAGE.

In vitro GTPase assay

GST-Rheb, GST-Ras, GST-Rac, and GST-Rap were expressed in the bacterial strain BL21 and purified using glutathione-Sepharose 4B beads (Sigma) as described. Small GTPases were loaded with ${ }^{32} \mathrm{P}-\gamma$-GTP before GAP assays. Here, $10 \mu \mathrm{g}$ of small GTPase protein bound on glutathione beads was incubated with $50 \mu \mathrm{Ci}$ of ${ }^{32} \mathrm{P}-\gamma$-GTP in loading buffer $(20 \mathrm{mM}$ Tris at $\mathrm{pH} 8,5 \mathrm{mM}$ EDTA, $1 \mathrm{mM}$ DTT, $0.1 \mathrm{mg} / \mathrm{mL} \mathrm{BSA}$ ) in a volume of $20 \mu \mathrm{L}$. The loading reaction was stopped by addition of $\mathrm{MgCl}_{2}$ to $10 \mathrm{mM}$. Then the small GTPase protein was eluted.

HA-TSC2 was transfected to HEK293 cells and immunoprecipitated by anti-HA antibody. The immune complexes were washed three times with wash buffer ( $20 \mathrm{mM}$ Tris at $\mathrm{pH} 7.4,800 \mathrm{mM} \mathrm{NaCl}, 2 \mathrm{mM}$ EDTA, $1 \%$ NP-40) and two times with GAP assay wash buffer $(20 \mathrm{mM}$ Tris at $\mathrm{pH} 8$, $100 \mathrm{mM} \mathrm{NaCl}, 5 \mathrm{mM} \mathrm{MgCl} 2,1 \mathrm{mM} \mathrm{DTT}$. The final GAP assays included $0.5 \mu \mathrm{g}$ of ${ }^{32} \mathrm{P}-\gamma$-GTP loaded small GTPase, and immunoprecipitated TSC2 from $1 \times 10^{7}$ transfected to HEK293 cells. Reactions were carried out in $40 \mu \mathrm{L}$ of GAP assay buffer $(20 \mathrm{mM}$ Tris at $\mathrm{pH} 8,10 \mathrm{mM}$ $\mathrm{MgCl}_{2}$, and $4 \mathrm{mM} \mathrm{DTT}$ ), at room temperature for $20 \mathrm{~min}$. The reaction was stopped by $300 \mu \mathrm{L}$ of charcoal buffer $(5 \%$ charcoal, $20 \mathrm{mM}$ phosphoric acid, $0.6 \mathrm{M} \mathrm{HCl}$ ), then vortexed for $10 \mathrm{~min}$ and spun at 13,000 rpm for $10 \mathrm{~min}$. Then $100 \mu \mathrm{L}$ of supernatant was taken, and the ${ }^{32} \mathrm{P}$ release was determined by scintillation counting.

In vivo labeling of Rheb

The cells were washed once with phosphate-free DMEM and incubated with $0.5 \mathrm{mCi} / \mathrm{mL}^{32} \mathrm{P}$-orthophosphate (ICN) for $4 \mathrm{~h}$. The cells were lysed with labeling lysis buffer (1\% Triton X-100, 50 mM HEPES at $\mathrm{pH} 7.4,100$ $\mathrm{mM} \mathrm{NaCl}, 5 \mathrm{mM} \mathrm{MgCl}$, $1 \mathrm{mg} / \mathrm{mL}$ BSA, $1 \mathrm{mM}$ DTT, $1 \mathrm{mM} \mathrm{PMSF}, 10$ $\mu \mathrm{g} / \mathrm{mL}$ leupeptin, $10 \mu \mathrm{g} / \mathrm{mL}$ aprotinin). Myc-tagged Rheb was immunoprecipitated. The Rheb-bound nucleotides were eluted with elution buffer ( $2 \mathrm{mM}$ EDTA, $0.2 \%$ SDS, $1 \mathrm{mM} \mathrm{GDP}, 1 \mathrm{mM} \mathrm{GTP}$ ) at $68^{\circ} \mathrm{C}$ for 20 $\mathrm{min}$. Then the eluted nucleotides were subjected to thin layer chromatography using PEI cellulose plates (Baker-flex) in $0.75 \mathrm{M} \mathrm{KH}_{2} \mathrm{PO}_{4}$ (pH 3.4).

\section{Acknowledgments}

We thank Tianqing Zhu for technical assistance. We also thank Weiquan $\mathrm{Li}$ and Huira Chong for plasmid construction and Haris G. Vikis and 
Jennifer Aurandt for critical reading of the manuscript. This work was supported by grants from the NIH and the Walther Cancer Institute.

The publication costs of this article were defrayed in part by payment of page charges. This article must therefore be hereby marked "advertisement" in accordance with 18 USC section 1734 solely to indicate this fact.

\section{References}

Au, K.S., Rodriguez, J.A., Finch, J.L., Volcik, K.A., Roach, E.S., Delgado, M.R., Rodriguez Jr., E., and Northrup, H. 1998. Germ-line mutational analysis of the TSC2 gene in 90 tuberous-sclerosis patients. Am. J. Hum. Genet. 62: 286-294.

Brown, E.J., Beal, P.A., Keith, C.T., Chen, J., Shin, T.B., and Schreiber, S.L. 1995. Control of p70 S6 kinase by kinase activity of FRAP in vivo. Nature 377: 441-446.

Clark, G.J., Kinch, M.S., Rogers-Graham, K., Sebti, S.M., Hamilton, A.D., and Der, C.J. 1997. The Ras-related protein Rheb is farnesylated and antagonizes Ras signaling and transformation. J. Biol. Chem. 272: 10608-10615.

Dennis, P.B., Jaeschke, A., Saitoh, M., Fowler, B., Kozma, S.C., and Thomas, G. 2001. Mammalian TOR: A homeostatic ATP sensor. Science 294: 1102-1105.

Desai, B.N., Myers, B.R., and Schreiber, S.L. 2002. FKBP12-rapamycinassociated protein associates with mitochondria and senses osmotic stress via mitochondrial dysfunction. Proc. Nat1. Acad. Sci. 99: 4319_ 4324.

The European Chromosome 16 Tuberous Sclerosis Consortium. 1993. Identification and characterization of the tuberous sclerosis gene on chromosome 16. Cell 75: 1305-1315.

Fang, Y., Vilella-Bach, M., Bachmann, R., Flanigan, A., and Chen, J. 2001. Phosphatidic acid-mediated mitogenic activation of mTOR signaling. Science 294: 1942-1945.

Gao, X. and Pan, D. 2001. TSC1 and TSC2 tumor suppressors antagonize insulin signaling in cell growth. Genes \& Dev. 15: 1383-1392.

Gao, X., Zhang, Y., Arrazola, P., Hino, O., Kobayashi, T., Yeung, R.S., Ru, B., and Pan, D. 2002. Tsc tumour suppressor proteins antagonize amino-acid-TOR signalling. Nat. Cell Biol. 4: 699-704.

Gingras, A.C., Raught, B., and Sonenberg, N. 1999. eIF4 initiation factors: Effectors of mRNA recruitment to ribosomes and regulators of translation. Annu. Rev. Biochem. 68: 913-963.

Gomez, M.R. 1991. Phenotypes of the tuberous sclerosis complex with a revision of diagnostic criteria. Ann. NY Acad. Sci. 615: 1-7.

Goncharova, E.A., Goncharov, D.A., Eszterhas, A., Hunter, D.S., Glassberg, M.K., Yeung, R.S., Walker, C.L., Noonan, D., Kwiatkowski, D.J., Chou, M.M., et al. 2002. Tuberin regulates p70 S6 kinase activation and ribosomal protein S6 phosphorylation. A role for the TSC2 tumor suppressor gene in pulmonary lymphangioleiomyomatosis (LAM). J. Biol. Chem. 277: 30958-30967.

Hara, K., Yonezawa, K., Weng, Q.P., Kozlowski, M.T., Belham, C., and Avruch, J. 1998. Amino acid sufficiency and mTOR regulate p70 S6 kinase and eIF-4E BP1 through a common effector mechanism. J. Biol. Chem. 273: 14484-14494.

Inoki, K., Li, Y., Zhu, T., Wu, J., and Guan, K.L. 2002. TSC2 is phosphorylated and inhibited by Akt and suppresses mTOR signalling. Nat. Cell Biol. 4: 648-657.

Jin, F., Wienecke, R., Xiao, G.H., Maize Jr., J.C., DeClue, J.E., and Yeung, R.S. 1996. Suppression of tumorigenicity by the wild-type tuberous sclerosis 2 (Tsc2) gene and its C-terminal region. Proc. Natl. Acad. Sci. 93: 9154-9159.

Kenerson, H.L., Aicher, L.D., True, L.D., and Yeung, R.S. 2002. Activated mammalian target of rapamycin pathway in the pathogenesis of tuberous sclerosis complex renal tumors. Cancer Res. 62: 5645-5650.

Kobayashi, T., Minowa, O., Sugitani, Y., Takai, S., Mitani, H., Kobayashi, E., Noda, T., and Hino, O. 2001. A germ-line Tsc1 mutation causes tumor development and embryonic lethality that are similar, but not identical to, those caused by Tsc2 mutation in mice. Proc. Natl. Acad. Sci. 98: 8762-8767.

Kwiatkowski, D.J. 2003. Tuberous sclerosis: From tubers to mTOR. Ann. Hum. Genet. 67: 87-96.

Lowy, D.R. and Willumsen, B.M. 1993. Function and regulation of ras. Annu. Rev. Biochem. 62: 851-891.

Mach, K.E., Furge, K.A., and Albright, C.F. 2000. Loss of Rhb1, a Rheb- related GTPase in fission yeast, causes growth arrest with a terminal phenotype similar to that caused by nitrogen starvation. Genetics 155: 611-622.

Manning, B.D., Tee, A.R., Logsdon, M.N., Blenis, J., and Cantley, L.C. 2002. Identification of the tuberous sclerosis complex-2 tumor suppressor gene product tuberin as a target of the phosphoinositide 3-kinase/akt pathway. Mol. Cell 10: 151-162.

Matsumoto, S., Bandyopadhyay, A., Kwiatkowski, D.J., Maitra, U., and Matsumoto, T. 2002. Role of the Tsc1-Tsc2 complex in signaling and transport across the cell membrane in the fission yeast Schizosaccharomyces pombe. Genetics 161: 1053-1063.

Momose, S., Kobayashi, T., Mitani, H., Hirabayashi, M., Ito, K., Ueda, M., Nabeshima, Y., and Hino, O. 2002. Identification of the coding sequences responsible for Tsc2-mediated tumor suppression using a transgenic rat system. Hum. Mol. Genet. 11: 2997-3006.

Potter, C.J. and Xu, T. 2001. Mechanisms of size control. Curr. Opin. Genet. Dev. 11: 279-286.

Potter, C.J., Huang, H., and Xu, T. 2001. Drosophila Tsc1 functions with Tsc2 to antagonize insulin signaling in regulating cell growth, cell proliferation, and organ size. Cell 105: 357-368.

Proud, C.G. 2002. Regulation of mammalian translation factors by nutrients. Eur. J. Biochem. 269: 5338-5349.

Scheffzek, K., Ahmadian, M.R., and Wittinghofer, A. 1998. GTPase-activating proteins: Helping hands to complement an active site. Trends Biochem. Sci. 23: 257-262.

Tapon, N., Ito, N., Dickson, B.J., Treisman, J.E., and Hariharan, I.K. 2001. The Drosophila tuberous sclerosis complex gene homologs restrict cell growth and cell proliferation. Cell 105: 345-355.

Tee, A.R., Fingar, D.C., Manning, B.D., Kwiatkowski, D.J., Cantley, L.C. and Blenis, J. 2002. Tuberous sclerosis complex-1 and complex-2 gene products function together to inhibit mammalian target of rapamycin (mTOR)-mediated downstream signaling. Proc. Natl. Acad. Sci. 99: 13571-13576.

Trahey, M. and McCormick, F. 1987. A cytoplasmic protein stimulates normal N-ras p21 GTPase, but does not affect oncogenic mutants. Science 238: 542-545.

Wienecke, R., Konig, A., and DeClue, J.E. 1995. Identification of tuberin, the tuberous sclerosis-2 product. Tuberin possesses specific RaplGAP activity. J. Biol. Chem. 270: 16409-16414.

Xiao, G.H., Shoarinejad, F., Jin, F., Golemis, E.A., and Yeung, R.S. 1997 The tuberous sclerosis 2 gene product, tuberin, functions as a Rab5 GTPase activating protein (GAP) in modulating endocytosis. J. Biol. Chem. 272: 6097-6100.

Yamagata, K., Sanders, L.K., Kaufmann, W.E., Yee, W., Barnes, C.A., Nathans, D., and Worley, P.F. 1994. rheb, a growth factor- and synaptic activity-regulated gene, encodes a novel Ras-related protein. $J$. Biol. Chem. 269: 16333-16339.

Yee, W.M. and Worley, P.F. 1997. Rheb interacts with Raf-1 kinase and may function to integrate growth factor- and protein kinase A-dependent signals. Mol. Cell. Biol. 17: 921-933. 


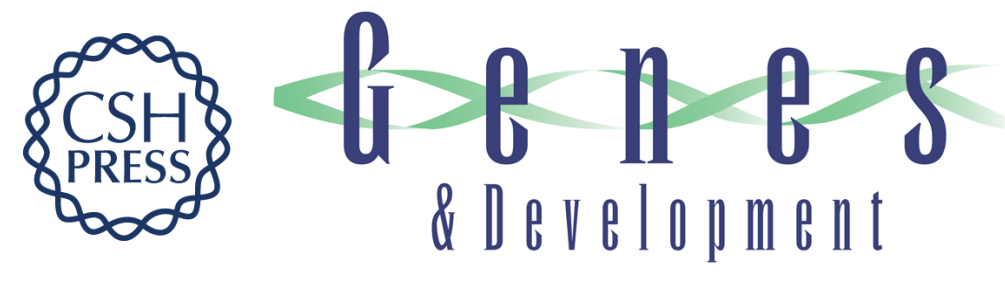

\section{Rheb GTPase is a direct target of TSC2 GAP activity and regulates mTOR signaling}

Ken Inoki, Yong Li, Tian Xu, et al.

Genes Dev. 2003, 17:

Access the most recent version at doi:10.1101/gad.1110003

References

This article cites 34 articles, 18 of which can be accessed free at: http://genesdev.cshlp.org/content/17/15/1829.full.html\#ref-list-1

License

Email Alerting

Receive free email alerts when new articles cite this article - sign up in the box at the top Service right corner of the article or click here.

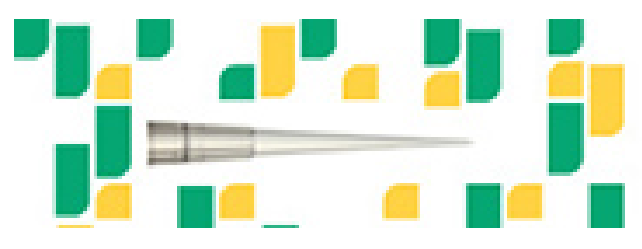

Focused on your science. 